\title{
Novel uses of fluorescent proteins
}

Alexander S. Mishin ${ }^{1,2}$, Vsevolod V. Belousov ${ }^{1}$, Kyril M. Solntsev ${ }^{3}$, Konstantin A. Lukyanov ${ }^{1,2}$

${ }^{1}$ Institute of Bioorganic Chemistry, Miklukho-Maklaya 16/10, 117997 Moscow, Russia

${ }^{2}$ Nizhny Novgorod State Medical Academy, Minin and Pozharsky Sq. 10/1, 603005 Nizhny Novgorod, Russia

${ }^{3}$ School of Chemistry and Biochemistry, Georgia Institute of Technology, 901 Atlantic Drive, Atlanta, Georgia 30332-0400, United States

Corresponding author: Lukyanov, Konstantin A (kluk@ibch.ru)

\begin{abstract}
The field of genetically encoded fluorescent probes is developing rapidly. New chromophore structures were characterized in proteins of Green Fluorescent Protein (GFP) family. A number of red fluorescent sensors, e.g., for $\mathrm{pH}, \mathrm{Ca}^{2+}$ and $\mathrm{H}_{2} \mathrm{O}_{2}$, were engineered for multiparameter imaging. Progress in development of microscopy hardware and software together with specially designed FPs pushed super-resolution fluorescence microscopy towards fast live-cell imaging. Deeper understanding of FPs structure and photophysics led to further development of imaging techniques. In addition to commonly used GFP-like proteins, unrelated types of FPs on the base of flavin-, bilirubin- or biliverdin-binding domains were designed. Their distinct biochemical and photophysical properties opened previously unexplored niches of FP uses such as labeling under anaerobic conditions, deep tissue imaging and even patients' blood analysis.
\end{abstract}




\section{Introduction}

In the broad instrumentarium of modern biology, fluorescent proteins occupy an important and unique niche enabling direct observation of molecular processes in live systems [1].

This technology began from a single known member, green fluorescent protein GFP from jellyfish Aequorea victoria. In 1990s, a number of its improved and color-shifted variants from blue to yellow were created. Since the discovery of GFP-like proteins in corals in 1999, great efforts were directed towards characterization of natural diversity of this protein family. As a result, GFP-like proteins were found not only in coelenterates, but also in combjellies, crustaceans, and lower chordates - lancelets [1]. Proteins of diverse spectral, biochemical and biophysical properties were found in nature leading to development of a multitude of FP-based techniques.

The interest in novel natural GFP-like proteins has diminished by now. The last notable finding in this field was a discovery of multidomain FPs in Hydrozoa in 2012 [2]. In these FPs, two or four GFP-like domains are repeated within the same polypeptide chain. Curiously, a linker between these domains contains amino acid sequence VAMPRIVET that means "hello to you" in Russian. This "best regards" from Nature should encourage scientists to further study biological functions and evolution diversity of this amazing protein family.

The focus of the GFP-like proteins research in the last few years has shifted towards deeper understanding of structure, photophysics and photochemistry of existing FPs and development of advanced imaging methods. An important new direction is engineering of FPs unrelated to GFP.

Applications of FPs are published in thousands of papers every year. In this short review, we focus only on a few topics, which in our opinion are of a special interest.

\section{From novel fluorescent proteins to novel applications}

In contrast to other natural proteinaceous pigments, which carry protein-bound cofactors, proteins of GFP family form chromophore by self-catalyzed posttranslational modifications of their own internal amino acids [1]. Different chromophores can be formed within the protein $\beta$ barrel (220-240 amino acids), and the list of known chromophore structures continues to expand (Fig. 1a). For example, an unusual linkage of GFP-type chromophore with the hydroxyl of a nearby tyrosine was recently revealed in red laRFP from a lancelet [3]. Also, in engineered green WasCFP a possibility to deprotonate the tryptophan-based CFP-type chromophore was demonstrated [4]. WasCFP possesses a record high fluorescence lifetime that is advantageous for multiparameter fluorescence lifetime imaging (FLIM) and fluorescence resonance energy transfer (FRET). Potentially, these new types of chromophores can facilitate further expansion of spectral diversity of GFP-like proteins.

Two-color labeling with regular FPs is a useful approach for quantitative imaging. For example, labeling of nascent mRNA with green and red FPs fused to specific RNA-binding domains was used to quantify kinetics of transcription of a single gene in live cells [5]. Also, artificial minigene constructs allow to use FP's green-to-red fluorescence ratio as a measure of activity of 
alternative splicing, mRNA translation or nonsense-mediated mRNA decay at single cell level [6-9]. Such analysis in some cases revealed strong heterogeneity within seemingly homogenous cell populations $[5,7,9]$ - phenomena that cannot be discovered by classical methods.

In addition to the GFP-like proteins, unrelated types of fluorescent proteins were designed during the last several years. These "genetically encoded" FPs are based on protein domains that specifically bind to ubiquitously present chromophores. The first representatives of such FPs were flavin mononucleotide (FMN)-based fluorescent proteins (FbFPs) developed in 2007 [10].

In FbFPs, light-oxygen-voltage-sensing (LOV) domains (about 100 amino acids) from bacterial or plant proteins are mutated to make the non-covalently bound FMN green fluorescent $[10,11]$ (Fig. 1b). The main advantages of FbFPs over GFP-like proteins are fast oxygen-independent maturation, which opens a whole area of applications under anaerobic conditions, and a smaller size, which was demonstrated to be crucial in some models. At the same time, compared to GFPs, FbFPs possess much lower fluorescence brightness because of low extinction coefficient.

An FbFP named miniSOG (Singlet Oxygen Generator) was designed to produce high level of reactive oxygen species (ROS) upon blue light illumination [12]. MiniSOG was found to be an efficient genetically encoded tag for electron microscopy. For live models, miniSOG and GFPlike protein KillerRed [13] (the latter produces radical-based ROS under green-orange light) can be used as genetically encoded photosensitizers. It is a unique tool that enables very precise (in space and time) light-induced killing of specific cell populations [13-17], inactivation of target proteins $\left[13,18^{*}, 19\right]$, and damaging genomic DNA [16,20]. Moreover, a new approach to study protein arrangement in large complexes was developed $\left[21^{* *}\right]$. This method named singlet oxygen triplet energy transfer (STET) evaluates distance between proteins using bleaching of infrared fluorescent protein IFP1.4 (fused to one target protein) by singlet oxygen produced by miniSOG (fused to another target protein). In contrast to commonly used FRET, which is efficient at short distances only $(<10 \mathrm{~nm})$, STET makes it possible to determine long-range (several tens of nanometres) inter-protein relationships. A recent appearance of an FbFP variant with enhanced level of singlet oxygen production [22] instils confidence in further development of phototoxic FP-based optogenetic techniques.

Another type of green FPs was discovered in Japanese eel muscles recently [23**]. This 139amino acid protein named UnaG belongs to the fatty acid binding protein (FABP) family. UnaG binds bilirubin non-covalently with a subnanomolar affinity and activates its fluorescence (Fig. 1c). Due to high affinity and specificity of bilirubin binding, UnaG can be used as a fluorescent sensor for the easy and fast measurement of bilirubin in blood of patients - the first case of suitability of a fluorescent protein for clinics [23**].

Engineered bacterial phytochromes represent the third type of non-GFP-like FPs [24]. These extensively mutated $\sim 315$ amino acids long domains carry covalently bound biliverdin that fluoresces in near-infrared (Fig. 1d). This spectral region (excitation-emission maxima at 640700 and $670-720 \mathrm{~nm}$, respectively) is unattainable for other types of FPs. The main drawback of bacteriophytochrome-based infrared FPs (IFPs) is low fluorescence quantum yield. Another 
problem of IFPs is insufficient amounts of biliverdin in some tissues [25]. This problem can be solved by co-expression of heme oxygenase, which produces biliverdin from heme [25].

In spite of low molecular brightness, IFPs far outperform GFP-like proteins in whole body imaging in mice, where near-infrared light penetrates tissues much deeper compared to shorterwavelength light $\left[26,27^{\star}\right]$. The use of a reversibly photoswitchable variants (PAiRFP1 and PAiRFP2) enables strong enhancement of signal-to-noise ratio by controlled optical modulation of the signal in in vivo imaging, where high autofluorescence represents a significant problem [28].

High extinction coefficient and low fluorescence quantum yield of IFPs are advantageous for photoacoustic tomography (PAT) [29]. PAT is a hybrid imaging technique, which is based on detection of ultrasound waves resulted from thermoelastic expansion of pigmented objects upon illumination with a short pulse of intense light. It was demonstrated that iRFP670 and iRFP720 [26] can be used for multiparameter PAT of deep tumors in mice, enabling clear spectral separation from each other and from blood hemoglobin [30*].

\section{Genetically encoded fluorescent biosensors go red}

One of the most powerful uses of GFP-like proteins is constructing genetically encoded fluorescent sensors for various analytes and protein activities [1]. Among different sensors' designs, perhaps the most promising one is based on circularly permuted FPs linked to the domain(s) that change conformation upon interaction with the molecule of interest. Green fluorescent proteins originated from Aequorea victoria GFP undergo circular permutation relatively easy. This resulted in many green fluorescent single-fluorophore sensors of superior performance for intracellular detection of $\mathrm{Ca}^{2+}$ [31], $\mathrm{NAD} / \mathrm{NADH}$ [32], ATP/ADP [33], $\mathrm{H}_{2} \mathrm{O}_{2}$ [34], etc. Sensors of other colors are in high demand for multiparameter imaging.

Recently, red fluorescent biosensors were developed. First, a number of red fluorescent $\mathrm{pH}$ sensors appeared [35-37] (Fig. 2a). With pHRed [35], quantitative imaging of pH changes is possible due to the ratiometric response. The probe has two excitation peaks at 440 and 585 $\mathrm{nm}$, and a single emission peak at $610 \mathrm{~nm}$. $\mathrm{p} K_{\mathrm{a}}$ of pHRed is 6.6 making it more suitable for $\mathrm{pH}$ measurements in neutral and slightly acidic compartments. But the sensor was used also to quantify mitochondrial $\mathrm{pH}$ changes. Later, two other red $\mathrm{pH}$ reporters appeared, pHTomato [36] and pHuji [37]. Both are intensiometric and used mostly to monitor endocytosis and exocytosis events. pHuji is based on mApple template and outperforms phTomato due to significantly higher dynamic range.

In 2011, a palette of GECOs-genetically encoded $\mathrm{Ca}^{2+}$ probes was reported [38], including the red one based on circularly permuted mApple FP (Fig. 2b). Later, other red fluorescent $\mathrm{Ca}^{2+}$ reporters appeared, namely RCaMP [39], R-CaMP1.07 [40], ratiometric REX-GEC01 [41], and low-affinity LAR-GECO for $\mathrm{Ca}^{2+}$ imaging in mitochondria and ER [42].

Whereas all existing green and red $\mathrm{Ca}^{2+}$ sensors detect $\mathrm{Ca}^{2+}$ transients, there is a need in a kind of "memory" indicator that would "remember" its $\mathrm{Ca}^{2+}$-bound state. This would allow whole-brain functional circuits labeling and analysis. Foscue et al. described a green-to-red photoconvertible $\mathrm{Ca}^{2+}$ sensor CaMPARI whose photoswitching efficiency strongly increases in the presence of 
$\mathrm{Ca}^{2+}\left[43^{* *}\right]$ (Fig. 2c). Thus, upon violet illumination only those neurons undergoing activitydependent $\mathrm{Ca}^{2+}$ elevation will turn red. The utility of the probe was demonstrated in a number of animal models ranging from fly to mouse.

cpmApple, a fluorophore of R-GECO1, was used to build HyPerRed, a genetically encoded red fluorescent $\mathrm{H}_{2} \mathrm{O}_{2}$ probe [44*]. HyPerRed enabled a first multiparameter imaging of mitochondrial $\mathrm{H}_{2} \mathrm{O}_{2}$ in parallel with glutathione redox state and $\mathrm{pH}$ in the mitochondrial matrix and in the cytoplasm imaged with green fluorescent biosensors, and also to monitor $\mathrm{H}_{2} \mathrm{O}_{2}$ dynamics in the mitochondria and in the cytoplasm of the same cells combining red and green probes for $\mathrm{H}_{2} \mathrm{O}_{2}$.

Most recently, a new design type for genetically encoded biosensors was suggested [45*]. It is based on green and red dimerization-dependent FPs that are fluorescent in the dimeric form and non-fluorescent as monomers. A number of biosensors, e.g., for caspase activity (Fig. 2c) and $\mathrm{Ca}^{2+}$, were built implementing an interchange of a non-fluorescent monomer between red and green dimers. Despite some limitations, such that non-quantitative readout, slow response and relatively low dynamic range, the dimerization-dependent biosensors provide a new useful way of probe design.

\section{Novel tags and approaches for super-resolution fluorescence microscopy}

The application of photoactivatable FPs (PAFPs) in two major types of super-resolution fluorescence microscopy - PALM (photoactivated localization microscopy) and RESOLFT (reversible saturatable optical fluorescence transition) was recently reviewed in Ref [46].

Despite the progress in methods and image reconstruction algorithms, we are still very far from the ultimate goal - prolonged live-cell super-resolution imaging at high temporal resolution. It should come as no surprise, that the certain single-molecule properties of fluorescent proteins (such as blinking behavior, emission rate, photoswitching rate, and the total photon budget) are essential for implementation of super-resolution techniques in living cells or tissues. For years, however, improvements of FPs was guided solely by ensemble properties, such as spectra, brightness, and photostability. Recently, the development of FPs placed emphasis on the specific requirements of super-resolution techniques. Recent works show comparison of singlemolecule behaviour of multiple PAFPs in fixed [47] and living cells [48].

In PALM and other similar techniques, super-resolution information is derived from localization of individual fluorophores, visible on image series as a result of controllable or stochastic singlemolecule switching. The requirement for spatial and temporal separation of fluorescence bursts significantly limits acquisition speed. An improvement in temporal resolution can be achieved by tuning of localization algorithms to novel sCMOS cameras with high frame rates [49]. Also, the progress in algorithms for more densely labelled samples brings the timescale of PALM closer to actual cellular dynamics [50]. Recently published mMaple2 and mMaple3 [51] were designed specifically to meet the requirements of PALM: high photon numbers per burst, optimal on-off switching rate ratio, reduced oligomerization tendency. The unavoidable intrinsic blinking of fluorescent proteins complicates single-molecule counting. A novel stochastic approach allows for extraction of accurate stoichiometry information from PALM data [52*]. 
Robust blinking of fluorophores can be advantageous, as in super-resolution optical fluctuation imaging (SOFI), a promising algorithm for densely labelled samples [53]. Recent extension of this method promises higher (up to 5-fold) resolution improvement and extraction of additional useful information from the fluctuations [54], such as molecular brightness. SOFI has been shown to work with PAFP $[55,56]$ even in $3 D\left[57^{* *}\right]$ in living cells. An improved reversibly switchable green FP Skylan-S was recently designed to provide robust fluctuations and low photofatigue for SOFI [58].

In RESOLFT, reversible photoswitching of fluorescent probes is utilized in coordinate-based system with structured nonlinear light beams. An elegant combination of RESOLFT and timedomain microscopy, dubbed $\square$-RESOLFT, allowed for dual-channel super-resolution imaging in living cells of spectrally similar rsEGFP and Dronpa with distinct fluorescent lifetimes and switching kinetics [59]. PAFPs for RESOLFT should also exhibit high switching rate and very low photofatigue over multiple cycles of photoconversion. The improvement of this property has already attracted researchers [60].

Correlative light and electron microscopy (CLEM) impose additional requirements on FPs. Most PAFPs lost fluorescence or the ability to photoactivation under conditions necessary for optimal ultrastructure fixation for EM. Recently published EosFP variants survive heavy $\mathrm{OsO}_{4}$ fixation, enabling better correlative PALM and electron microscopy [61].

All of these techniques are rapidly improving towards implementation in vivo. One recent example is the observation of presence of caveolae membrane domains in a living zebrafish embryo [62].

\section{Identification and utilization of the long-lived dark states in fluorescent proteins}

All FPs have fluorescence quantum yields lower than unity, indicating the presence of nonradiative processes competing with fluorescence. In some cases, such non-radiative processes may involve the formation of kinetically trapped dark states (DS) of various origins. The formation and utilization of such DS are largely unexplored, and this section will discuss few recent achievements in this area.

Dickson et al. proposed the utilization of DS using the principles of modulated spectroscopy [62]. The simplified Jablonski diagram of the suggested approach is shown in Figure 3a. A direct depopulation of transient DS by coillumination of the sample with secondary laser was suggested. This will lead to repopulation of the chromophore emitting singlet excited state enhancing its fluorescence. Thus the crucial requirement for optically enhanced fluorescence is to have a relatively long lived and the bathochromically shifted optically reversible dark state. One of obvious advantages of this method is that photon energy of the secondary light source $\left(h v_{3}\right)$ is less than that of the primarily $S_{0} \rightarrow S_{1}$ excitation $\left(h v_{1}\right)$ and the fluorescence $\left(h v_{2}\right)$. Turning this coillumination on and off at a specific frequency dynamically modulates collected fluorescence without generating additional background. This method, initially developed and utilized for metal nanodots and organic dyes, has been recently applied to FPs. Some blue (omBFP, the variants of mKalama1), green (AcGFP), red, and other FPs were susceptible to secondary pulsed excitation [64-66]. One impressive example includes the fluorescence 
recovery of the green FP in the presence of highly fluorescent, but unmodulatable another green FP (Figure 3b). Another example demonstrates a dramatic improvement of signal-to-noise ratio for the blue FP (Figure 3c).

Using the broadband transient absorption (TA) spectroscopy spanning the time scale from picoseconds to seconds, a hidden photoinduced reactivity of various FPs and the nature of the DS can be revealed. Figure $3 d$ shows the TA spectra of selected modulatable FPs in comparison to their DS action spectra. In case of bright blue-light emitting protein omBFP, the DS transient was associated with the anionic species, which are formed as a result of isomerization-coupled internal conversion and deprotonation $[67,68]$. The unpublished DS action spectrum of mDsRed had a maximum around $710 \mathrm{~nm}$ (see Fig. 5.4 in Ref. [66]), which nicely fits the TA spectra of various RFPs [69*]. Based on the results of QM/MM calculations this transient was assigned to the unusual open-shell dianionic chromophore (dianion-radical) formed via photoreduction. The results raise the question of whether photoreduction occurs in other FPs and if the corresponding electron-attached states are common gateway states for photobleaching and blinking. The nature of the DS in AcGFP is unclear at the moment.

We believe that previously unexplored arena of FP DS holds great promise for future understanding, development, and utilization of FPs.

\section{Conclusions}

At first glance, applications of fluorescent proteins (FPs) seem to be so well-established that nothing really new can appear in this field, but recent works convince of the opposite. Directed molecular evolution is extensively used to generate GFP-like proteins with new chromophores, unusual spectra, extended fluorescence lifetime, enhanced photostability, improved parameters of photoconversion, etc. Another important growing point is construction of new biosensors, in particular red fluorescent ones. The use of sensors of distinct colors makes it possible to simultaneously observe various analytes and activities within the same cell.

GFP-unrelated FPs based on protein domains bound to natural intracellular cofactors (FMN, bilirubin or biliverdin) are of a special interest. These FPs are seemingly "genetically encoded" since there is no need to introduce exogenous dyes into the cells. Non-GFP-like FPs possess unique properties such as oxygen-independent maturation, near infrared fluorescence, or efficient ROS production, which enable novel areas of applications where GFP-like proteins are inefficient.

Novel improved FPs are envisioned for further improvement of quantitative multiparameter imaging of live systems at different scales, from single molecules to whole organisms.

\section{Acknowledgements}

We are indebted to all collaborators listed in joint publications. Financial support from the Russian Science Foundation (grant 14-25-00129 to K.A.L.) and National Science Foundation (grant CHE-1213047 to K.M.S.) is greatly acknowledged. 


\section{Figure legends}

Figure 1. Structures of fluorescent proteins of different types. 3D protein structures are shown to the same scale in cartoon representation (rainbow colors from blue at $\mathrm{N}$-termini to red at $\mathrm{C}$ termini). Chromophore groups are shown in spacefill representation. Images were created using PyMol (DeLano Scientific) from PDB files 2Y0G, 4EET, 4I3B, and 1ZTU for (a-d), respectively. In chemical structures of chromophores the moieties responsible for fluorescence are highlighted by corresponding colors. (a) GFP-like proteins. Main types of chromophores formed by autocatalytic modifications of internal amino acids are shown. (b) FMN-binding fluorescent proteins (PpFbFP, iLOV, miniSOG, etc.). (c) Bilirubin-binding protein UnaG. (d) Biliverdinbinding fluorescent proteins (IFP1.4, iRFP, IFP2.0, etc.).

Figure 2. Design of FP-based sensors. Selected sensor types discussed in the review are shown. (a) Single FP-based $\mathrm{pH}$ sensors, which increase red fluorescence at higher $\mathrm{pH}$. (b) Calcium sensors based on circularly permuted red FPs fused to M13 peptide and calmodulin (CaM), which increase red fluorescence in $\mathrm{Ca}^{2+}$-bound state. (c) $\mathrm{Ca}^{2+}$ sensor CaMPARI. Its green-to-red photoswitching under violet light illumination occurs efficiently only from the $\mathrm{Ca}^{2+}$ bound state. (d) Sensor for caspase-3 activity based on dimerization-dependent green and red FPs, which become brightly fluorescent only upon binding with a non-fluorescent monomer (grey).

Figure 3. Dark states in action. (a) Jablonski diagram illustrating the reverse intersystem crossing between bright and dark states of a fluorophore. Note that this scheme works for FPs and other systems which depopulates DS and repopulates $S_{0}$. For some other systems the direct repopulation of $S_{1}$ is possible. (b) Selective fluorescence recovery of mitochondriatargeted AcGFP in the presence of high nuclear-targeted EGFP fluorescence for live NIH 3T3 cells: left image - raw fluorescence of AcGFP-labeled mitochondria and EGFP excited at 476 $\mathrm{nm}$; right image - demodulated AcGFP fluorescence upon coexcitation with $561 \mathrm{~nm} 300 \mathrm{~Hz}$ laser. (Reproduced with permission from ref [65] which include all experimental details. Copyright 2012 American Chemical Society). (c) Live-cell demodulation of mitochondriatargeted modBFP/H148K. Upon $405 \mathrm{~nm}$ illumination, blue fluorescence was collected from modBFP/H148K-mito mixed with high background emission. Coillumination at $514.5 \mathrm{~nm}$ modulated at $2 \mathrm{~Hz}$ (secondary illumination only within the white circle) recovered only the modBFP/H148K-mito signal on a greatly reduced background (lower circle). (Reproduced with permission from ref [63]. Copyright 2013 American Chemical Society. See the original reference for the experimental details). (d) Optical properties of the modulatable FPs. Normalized absorption (blue lines) and emission (green lines) spectra, as well as transient absorption spectra (magenta lines) recorded at $4 \mathrm{~ms}$ after laser excitation. DS action cross section spectra scanned by secondary laser wavelength of various FPs (red squares). modBFP was excited at $372 \mathrm{~nm}$, AcGFP at $476 \mathrm{~nm}$. TA of DsRed is presented. 


\section{References}

1. Chudakov DM, Matz MV, Lukyanov S, Lukyanov KA: Fluorescent proteins and their applications in imaging living cells and tissues. Physiol. Rev. 2010, 90:1103-1163.

2. Hunt ME, Modi CK, Aglyamova GV, Ravikant DVS, Meyer E, Matz MV: Multi-domain GFPlike proteins from two species of marine hydrozoans. Photochem. Photobiol. Sci. 2012, 11:637-644.

3. Pletnev VZ, Pletneva NV, Lukyanov KA, Souslova EA, Fradkov AF, Chudakov DM, Chepurnykh T, Yampolsky IV, Wlodawer A, Dauter Z, et al:: Structure of the red fluorescent protein from a lancelet (Branchiostoma lanceolatum): a novel GYG chromophore covalently bound to a nearby tyrosine. Acta Crystallogr. D Biol. Crystallogr. 2013, 69:1850-1860.

4. Sarkisyan KS, Yampolsky IV, Solntsev KM, Lukyanov SA, Lukyanov KA, Mishin AS: Tryptophan-based chromophore in fluorescent proteins can be anionic. Sci. Rep. 2012, 2:608.

5. Hocine S, Raymond P, Zenklusen D, Chao JA, Singer RH: Single-molecule analysis of gene expression using two-color RNA labeling in live yeast. Nat. Methods 2013, 10:119-121.

6. Dean KM, Grayhack EJ: RNA-ID, a highly sensitive and robust method to identify cisregulatory sequences using superfolder GFP and a fluorescence-based assay. $R N A$ 2012, 18:2335-2344.

7. Gurskaya NG, Staroverov DB, Zhang L, Fradkov AF, Markina NM, Pereverzev AP, Lukyanov KA: Analysis of alternative splicing of cassette exons at single-cell level using two fluorescent proteins. Nucleic Acids Res. 2012, 40:e57.

8. Zheng S, Damoiseaux R, Chen L, Black DL: A broadly applicable high-throughput screening strategy identifies new regulators of Dlg4 (Psd-95) alternative splicing. Genome Res. 2013, 23:998-1007.

9. Pereverzev AP, Gurskaya NG, Ermakova GV, Kudryavtseva El, Markina NM, Kotlobay AA, Lukyanov SA, Zaraisky AG, Lukyanov KA: Method for quantitative analysis of nonsense-mediated mRNA decay at the single cell level. Sci. Rep. 2015, 5:7729.

10. Drepper T, Eggert T, Circolone F, Heck A, Krauss U, Guterl J-K, Wendorff M, Losi A, Gärtner W, Jaeger K-E: Reporter proteins for in vivo fluorescence without oxygen. Nat. Biotechnol. 2007, 25:443-445.

11. Mukherjee A, Weyant KB, Agrawal U, Walker J, Cann IKO, Schroeder CM: Engineering and characterization of new LOV-based fluorescent proteins from Chlamydomonas reinhardtii and Vaucheria frigida. ACS Synth. Biol. $2014 \mathrm{http}: / / \mathrm{dx} . d o i . o r g /$ 10.1021/sb500237x.

12. Shu X, Lev-Ram V, Deerinck TJ, Qi Y, Ramko EB, Davidson MW, Jin Y, Ellisman MH, Tsien RY: A genetically encoded tag for correlated light and electron microscopy of intact cells, tissues, and organisms. PLOS Biol. 2011, 9:e1001041.

13. Bulina ME, Chudakov DM, Britanova OV, Yanushevich YG, Staroverov DB, Chepurnykh TV, Merzlyak EM, Shkrob MA, Lukyanov S, Lukyanov KA: A genetically encoded photosensitizer. Nat. Biotechnol. 2006, 24:95-99.

14. Qi YB, Garren EJ, Shu X, Tsien RY, Jin Y: Photo-inducible cell ablation in Caenorhabditis elegans using the genetically encoded singlet oxygen generating protein miniSOG. Proc. Natl. Acad. Sci. U. S. A. 2012, 109:7499-7504. 
15. Williams DC, Bejjani RE, Ramirez PM, Coakley S, Kim SA, Lee $H$, Wen Q, Samuel A, Lu $H$, Hilliard MA et al.: Rapid and permanent neuronal inactivation in vivo via subcellular generation of reactive oxygen with the use of KillerRed. Cell Rep. 2013, 5:553-563.

16. Ryumina AP, Serebrovskaya EO, Shirmanova MV, Snopova LB, Kuznetsova MM, Turchin IV, Ignatova NI, Klementieva NV, Fradkov AF, Shakhov BE et al.: Flavoprotein miniSOG as a genetically encoded photosensitizer for cancer cells. Biochim. Biophys. Acta 2013, 1830:5059-5067.

17. Kuznetsova DS, Shirmanova MV, Dudenkova VV, Subochev PV, Turchin IV, Zagaynova EV, Lukyanov SA, Shakhov BE, Kamensky VA: Photobleaching and phototoxicity of KillerRed in tumor spheroids induced by continuous wave and pulsed laser illumination. J. Biophotonics 2015, doi: 10.1002/jbio.201400130.

18. * Takemoto K, Matsuda T, Sakai N, Fu D, Noda M, Uchiyama S, Kotera I, Arai Y, Horiuchi $\mathrm{M}$, Fukui $\mathrm{K}$ et al.: SuperNova, a monomeric photosensitizing fluorescent protein for chromophore-assisted light inactivation. Sci. Rep. 2013, 3:2629.

Authors developed a monomeric variant of KillerRed, which is potentially widely applicable for light-induced inactivation of target proteins.

19. Lin JY, Sann SB, Zhou K, Nabavi S, Proulx CD, Malinow R, Jin Y, Tsien RY: Optogenetic inhibition of synaptic release with chromophore-assisted light inactivation (CALI). Neuron 2013, 79:241-253.

20. Lan L, Nakajima S, Wei L, Sun L, Hsieh C-L, Sobol RW, Bruchez M, Van Houten B, Yasui A, Levine AS: Novel method for site-specific induction of oxidative DNA damage reveals differences in recruitment of repair proteins to heterochromatin and euchromatin. Nucleic Acids Res. 2014, 42:2330-2345.

21. ${ }^{* *}$ To T-L, Fadul MJ, Shu X: Singlet oxygen triplet energy transfer-based imaging technology for mapping protein-protein proximity in intact cells. Nat. Commun. 2014, 5:4072.

A principally new approach to decipher mutual arrangement of proteins in large complexes was developed.

22. Torra J, Burgos-Caminal A, Endres S, Wingen M, Drepper T, Gensch T, Ruiz-González R, Nonell S: Singlet oxygen photosensitisation by the fluorescent protein Pp2FbFP L30M, a novel derivative of Pseudomonas putida flavin-binding Pp2FbFP. Photochem. Photobiol. Sci. 2015, 14:280-287.

23. ${ }^{* *}$ Kumagai A, Ando R, Miyatake H, Greimel P, Kobayashi T, Hirabayashi $\mathrm{Y}$, Shimogori T, Miyawaki A: A bilirubin-inducible fluorescent protein from eel muscle. Cell 2013, 153:1602-1611.

The first representative of new family of bilirubin-binding fluorescent proteins was discovered.

This brightly fluorescent protein with oxygen-independent maturation appears to be a promising tag for basic biology and even for clinical analyses.

24. Piatkevich KD, Subach FV, Verkhusha VV: Engineering of bacterial phytochromes for near-infrared imaging, sensing, and light-control in mammals. Chem. Soc. Rev. 2013, 42:3441-3452.

25. Yu D, Gustafson WC, Han C, Lafaye C, Noirclerc-Savoye M, Ge W-P, Thayer DA, Huang $H$, Kornberg TB, Royant A et al:: An improved monomeric infrared fluorescent protein for neuronal and tumour brain imaging. Nat. Commun. 2014, 5:3626.

26. Shcherbakova DM, Verkhusha VV: Near-infrared fluorescent proteins for multicolor in vivo imaging. Nat. Methods 2013, 10:751-754. 
27. * Rice WL, Shcherbakova DM, Verkhusha VV, Kumar ATN: In vivo tomographic imaging of deep seated cancer using fluorescence lifetime contrast. Cancer Res. 2015, doi:10.1158/0008-5472.CAN-14-3001.

Fluorescence lifetime imaging enabled three-color detection of IFPs-expressing tumors deep in mouse tissues.

28. Piatkevich KD, Subach FV, Verkhusha VV: Far-red light photoactivatable near-infrared fluorescent proteins engineered from a bacterial phytochrome. Nat. Commun. 2013, 4:2153.

29. Filonov GS, Krumholz A, Xia J, Yao J, Wang LV, Verkhusha VV: Deep-tissue photoacoustic tomography of a genetically encoded near-infrared fluorescent probe. Angew. Chem. Int. Ed Engl. 2012, 51:1448-1451.

30. * Krumholz A, Shcherbakova DM, Xia J, Wang LV, Verkhusha VV: Multicontrast photoacoustic in vivo imaging using near-infrared fluorescent proteins. Sci. Rep. 2014, 4:3939.

It was demonstrated that iRFP670 and iRFP720 can be nicely distinguished from each other and from hemoglobin by photoacoustic tomography in the tumor-bearing mice model.

31. Chen T-W, Wardill TJ, Sun Y, Pulver SR, Renninger SL, Baohan A, Schreiter ER, Kerr RA, Orger MB, Jayaraman $\mathrm{V}$, et al.: Ultrasensitive fluorescent proteins for imaging neuronal activity. Nature 2013, 499:295-300.

32. Hung YP, Albeck JG, Tantama M, Yellen G: Imaging cytosolic NADH-NAD(+) redox state with a genetically encoded fluorescent biosensor. Cell Metab. 2011, 14:545-554.

33. Tantama M, Martínez-François JR, Mongeon R, Yellen G: Imaging energy status in live cells with a fluorescent biosensor of the intracellular ATP-to-ADP ratio. Nat. Commun. 2013, 4:2550.

34. Bilan DS, Pase L, Joosen L, Gorokhovatsky AY, Ermakova YG, Gadella TWJ, Grabher C, Schultz C, Lukyanov S, Belousov VV: HyPer-3: A genetically encoded $\mathrm{H}_{2} \mathrm{O}_{2}$ probe with improved performance for ratiometric and fluorescence lifetime imaging. ACS Chem. Biol. 2013, 8:535-542.

35. Tantama M, Hung YP, Yellen G: Imaging intracellular pH in live cells with a genetically encoded red fluorescent protein sensor. J. Am. Chem. Soc. 2011, 133:10034-10037.

36. Li Y, Tsien RW: pHTomato, a red, genetically encoded indicator that enables multiplex interrogation of synaptic activity. Nat. Neurosci. 2012, 15:1047-1053.

37. Shen $\mathrm{Y}$, Rosendale M, Campbell RE, Perrais D: pHuji, a pH-sensitive red fluorescent protein for imaging of exo- and endocytosis. J. Cell Biol. 2014, 207:419-432.

38. Zhao Y, Araki S, Wu J, Teramoto T, Chang Y-F, Nakano M, Abdelfattah AS, Fujiwara M, Ishihara T, Nagai $\mathrm{T}$ et al:: An expanded palette of genetically encoded $\mathrm{Ca2+}$ indicators. Science 2011, 333:1888-1891.

39. Akerboom J, Carreras Calderón N, Tian L, Wabnig S, Prigge M, Tolö J, Gordus A, Orger $\mathrm{MB}$, Severi KE, Macklin JJ et al.: Genetically encoded calcium indicators for multi-color neural activity imaging and combination with optogenetics. Front. Mol. Neurosci. 2013, 6:2.

40. Ohkura M, Sasaki T, Kobayashi C, Ikegaya Y, Nakai J: An improved genetically encoded red fluorescent $\mathrm{Ca2}+$ indicator for detecting optically evoked action potentials. PLOS One 2012, 7:e39933.

41. Wu J, Abdelfattah AS, Miraucourt LS, Kutsarova E, Ruangkittisakul A, Zhou H, Ballanyi K, 
Wicks G, Drobizhev M, Rebane A et al:: A long Stokes shift red fluorescent Ca2+ indicator protein for two-photon and ratiometric imaging. Nat. Commun. 2014, 5:5262.

42. Wu J, Prole DL, Shen Y, Lin Z, Gnanasekaran A, Liu Y, Chen L, Zhou H, Chen SRW, Usachev YM et al:: Red fluorescent genetically encoded $\mathrm{Ca}^{2+}$ indicators for use in mitochondria and endoplasmic reticulum. Biochem. J 2014, 464:13-22.

43. ** Fosque BF, Sun Y, Dana H, Yang C-T, Ohyama T, Tadross MR, Patel R, Zlatic M, Kim DS, Ahrens MB et al.: Neural circuits. Labeling of active neural circuits in vivo with designed calcium integrators. Science 2015, 347:755-760.

Authors describe $\mathrm{Ca}^{2+}$ "memory" sensor CaMPARI based on a photoswitchable FP, in which green-to-red photoconversion efficiency depends on $\mathrm{Ca}^{2+}$ binding.

44. * Ermakova YG, Bilan DS, Matlashov ME, Mishina NM, Markvicheva KN, Subach OM, Subach FV, Bogeski I, Hoth M, Enikolopov G et al.: Red fluorescent genetically encoded indicator for intracellular hydrogen peroxide. Nat. Commun. 2014, 5:5222.

The paper describes the first non-calcium red fluorescent indicator based on a circularly permuted RFP.

45. * Ding Y, Li J, Enterina JR, Shen Y, Zhang I, Tewson PH, Mo GCH, Zhang J, Quinn AM, Hughes TE et al:: Ratiometric biosensors based on dimerization-dependent fluorescent protein exchange. Nat. Methods 2015, doi:10.1038/nmeth.3261.

The paper presents a novel principle of design of genetically encoded sensors.

46. Shcherbakova DM, Sengupta P, Lippincott-Schwartz J, Verkhusha VV: Photocontrollable fluorescent proteins for superresolution imaging. Annu. Rev. Biophys. 2014, 43:303329.

47. Avilov S, Berardozzi R, Gunewardene MS, Adam V, Hess ST, Bourgeois D: In cellulo evaluation of phototransformation quantum yields in fluorescent proteins used as markers for single-molecule localization microscopy. PLoS One 2014, 9:e98362.

48. Durisic N, Laparra-Cuervo L, Sandoval-Álvarez A, Borbely JS, Lakadamyali M: Singlemolecule evaluation of fluorescent protein photoactivation efficiency using an in vivo nanotemplate. Nat. Methods 2014, 11:156-162.

49. Huang F, Hartwich TMP, Rivera-Molina FE, Lin Y, Duim WC, Long JJ, Uchil PD, Myers JR, Baird MA, Mothes W et al.: Video-rate nanoscopy using sCMOS camera-specific single-molecule localization algorithms. Nat. Methods 2013, 10:653-658.

50. Min J, Vonesch C, Kirshner H, Carlini L, Olivier N, Holden S, Manley S, Ye JC, Unser M: FALCON: fast and unbiased reconstruction of high-density super-resolution microscopy data. Sci. Rep. 2014, 4:4577.

51. Wang S, Moffitt JR, Dempsey GT, Xie XS, Zhuang X: Characterization and development of photoactivatable fluorescent proteins for single-molecule-based superresolution imaging. Proc. Natl. Acad. Sci. U. S. A. 2014, 111:8452-8457.

52. * Rollins GC, Shin JY, Bustamante C, Pressé S: Stochastic approach to the molecular counting problem in superresolution microscopy. Proc. Natl. Acad. Sci. U. S. A. 2015, 112:E110-8.

The paper provides a novel approach to extract molecular counts from PALM data.

53. Dertinger T, Colyer R, lyer G, Weiss S, Enderlein J: Fast, background-free, 3D superresolution optical fluctuation imaging (SOFI). Proc. Natl. Acad. Sci. U. S. A. 2009, 106:22287-22292.

54. Geissbuehler S, Bocchio N, Dellagiacoma C, Berclaz C, Leutenegger M, Lasser T: 
Mapping molecular statistics with balanced super-resolution optical fluctuation imaging (bSOFI). Optical Nanoscopy 2012, 1:4.

55. Dedecker $\mathrm{P}$, Mo GCH, Dertinger $\mathrm{T}$, Zhang J: Widely accessible method for superresolution fluorescence imaging of living systems. Proc. Natl. Acad. Sci. U. S. A. 2012, 109:10909-10914.

56. Moeyaert B, Nguyen Bich N, De Zitter E, Rocha S, Clays K, Mizuno H, van Meervelt L, Hofkens J, Dedecker P: Green-to-red photoconvertible Dronpa mutant for multimodal super-resolution fluorescence microscopy. ACS Nano 2014, 8:1664-1673.

57. ** Geissbuehler S, Sharipov A, Godinat A, Bocchio NL, Sandoz PA, Huss A, Jensen NA, Jakobs S, Enderlein J, Gisou van der Goot $F$ et al.: Live-cell multiplane threedimensional super-resolution optical fluctuation imaging. Nat. Commun. 2014, 5:5830. The paper shows multiplane 3D SOFI of living cells labelled with reversibly switchable fluorescent protein with short acquisition time.

58. Zhang X, Chen X, Zeng Z, Zhang M, Sun Y, Xi P, Peng J, Xu P: Development of a reversibly switchable fluorescent protein for super-resolution optical fluctuation imaging (SOFI). ACS Nano 2015, doi:10.1021/nn5064387.

59. Testa I, D'Este E, Urban NT, Balzarotti F, Hell SW: Dual channel RESOLFT nanoscopy by using fluorescent state kinetics. Nano Lett. 2015, 15:103-106.

60. Duan C, Byrdin M, Kathib ME, Henry X, Adam V, Bourgeois D: Rational design of enhanced photoresistance in a photoswitchable fluorescent protein. Methods and Applications in Fluorescence 2015, 3:014004.

61. Paez-Segala MG, Sun MG, Shtengel G, Viswanathan S, Baird MA, Macklin JJ, Patel R, Allen JR, Howe ES, Piszczek G et al.: Fixation-resistant photoactivatable fluorescent proteins for CLEM. Nat. Methods 2015, 12:215-218.

62. Gabor KA, Kim D, Kim CH, Hess ST: Nanoscale imaging of caveolin-1 membrane domains in vivo. PLoS One 2015, 10:e0117225.

63. Hsiang J-C, Jablonski AE, Dickson RM: Optically modulated fluorescence bioimaging: visualizing obscured fluorophores in high background. Acc. Chem. Res. 2014, 47:1545-1554.

64. Jablonski AE, Vegh RB, Hsiang J-C, Bommarius B, Chen Y-C, Solntsev KM, Bommarius AS, Tolbert LM, Dickson RM: Optically modulatable blue fluorescent proteins. J. Am. Chem. Soc. 2013, 135:16410-16417.

65. Jablonski AE, Hsiang J-C, Bagchi P, Hull N, Richards CI, Fahrni CJ, Dickson RM: Signal discrimination between fluorescent proteins in live cells by long-wavelength optical modulation. J. Phys. Chem. Lett. 2012, 3:3585-3591.

66. Jablonski $A E$ : Optically modulated fluorescent proteins. Dissertation. Georgia Institute of Technology. 2014. URI: http://hdl.handle.net/1853/52327.

67. Vegh RB, Bloch DA, Bommarius AS, Iwaï H, Pletnev SV, Verkhovsky M, Bochenkova AV, Solntsev KM: Hidden photoinduced reactivity in the blue fluorescent protein mKalama1. Submitted.

68. Vegh R: Mechanisms and applications of photoinduced processes in fluorescent proteins. Dissertation. Georgia Institute of Technology. 2012. URI: http://hdl.handle.net/1853/50126.

69. * Vegh RB, Bravaya KB, Bloch DA, Bommarius AS, Tolbert LM, Verkhovsky M, Krylov Al, 
Solntsev KM: Chromophore photoreduction in red fluorescent proteins is responsible for bleaching and phototoxicity. J. Phys. Chem. B 2014, 118:4527-4534.

First example of broadband TA spectroscopy in FPs. Identification of the DS using QM/MM methods. 
Figures

Figure 1

(a)
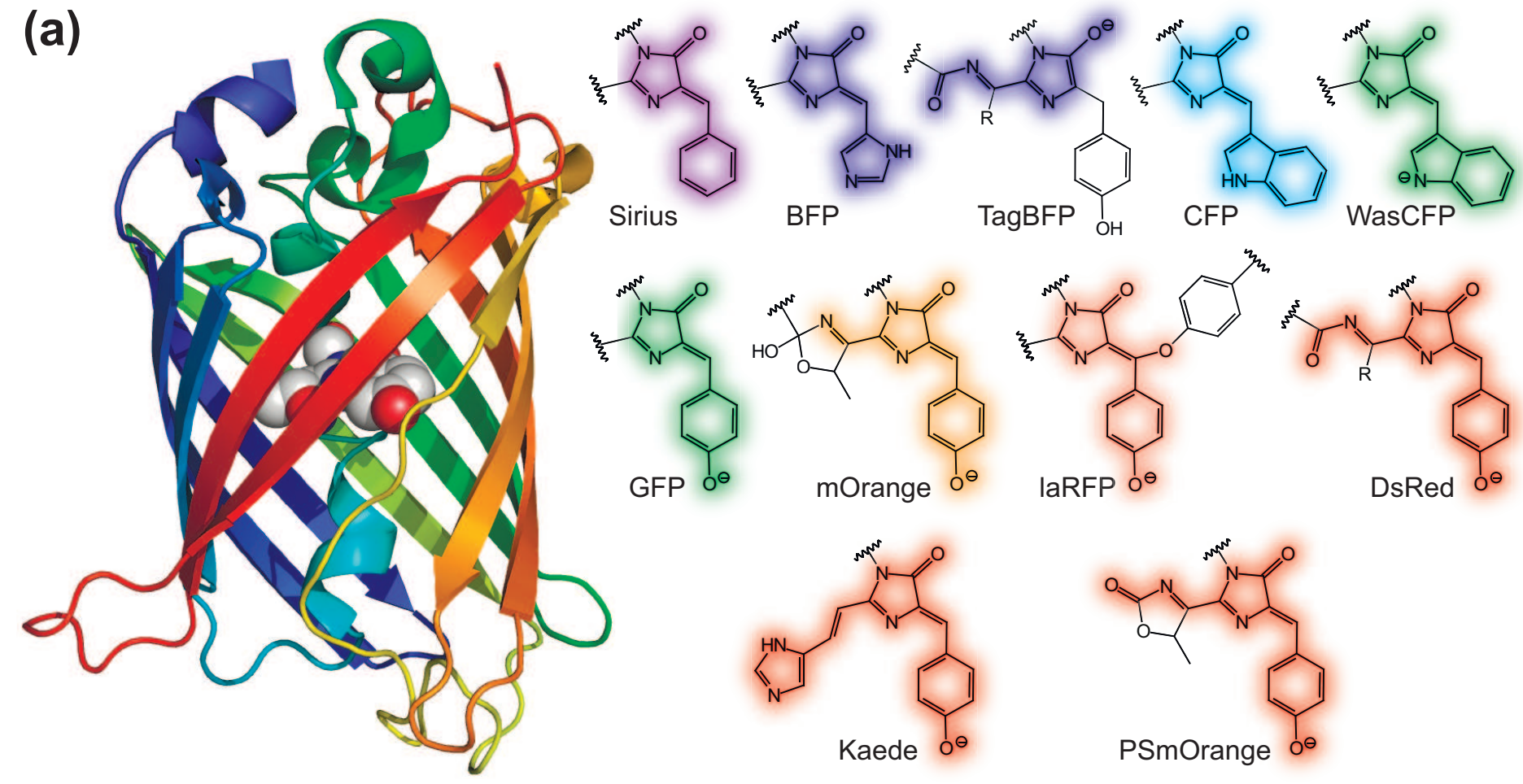

(b)
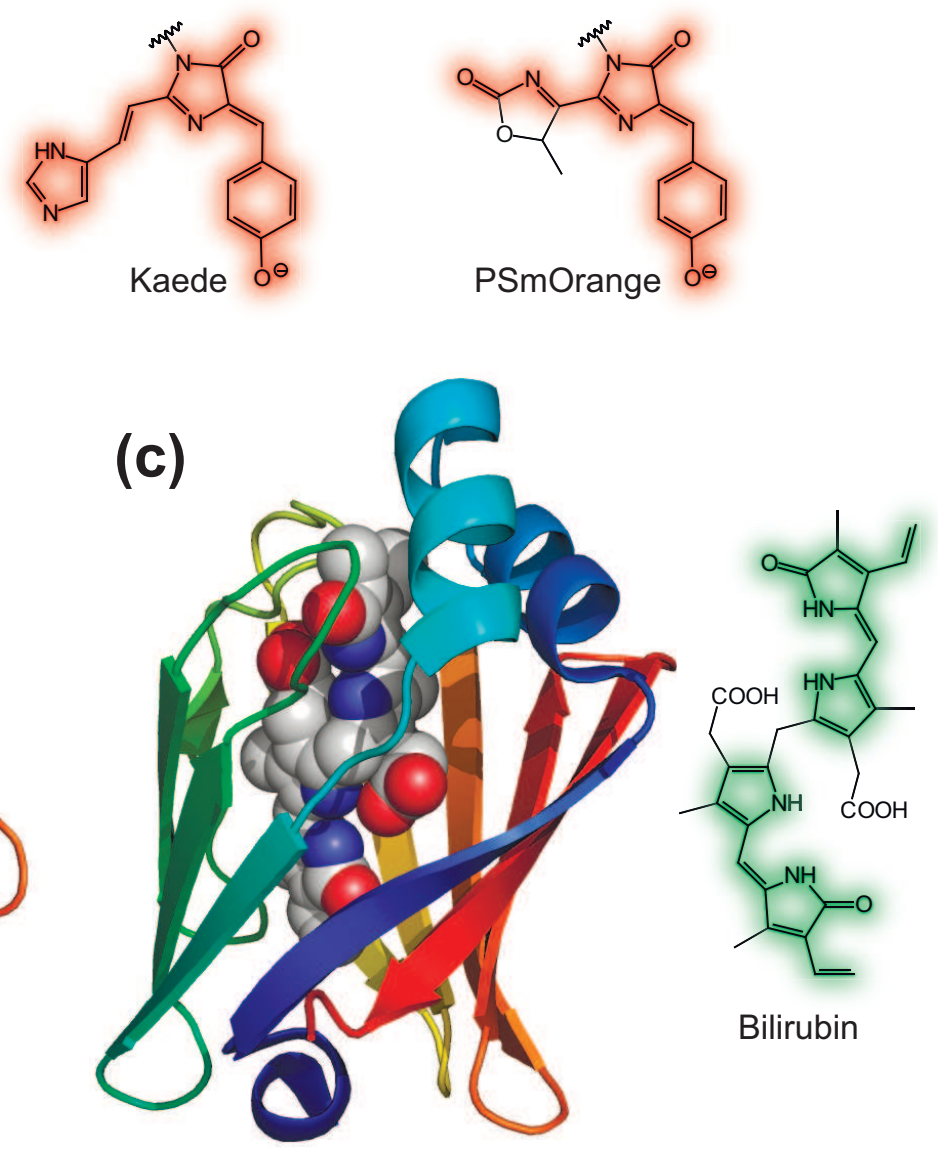

(d)

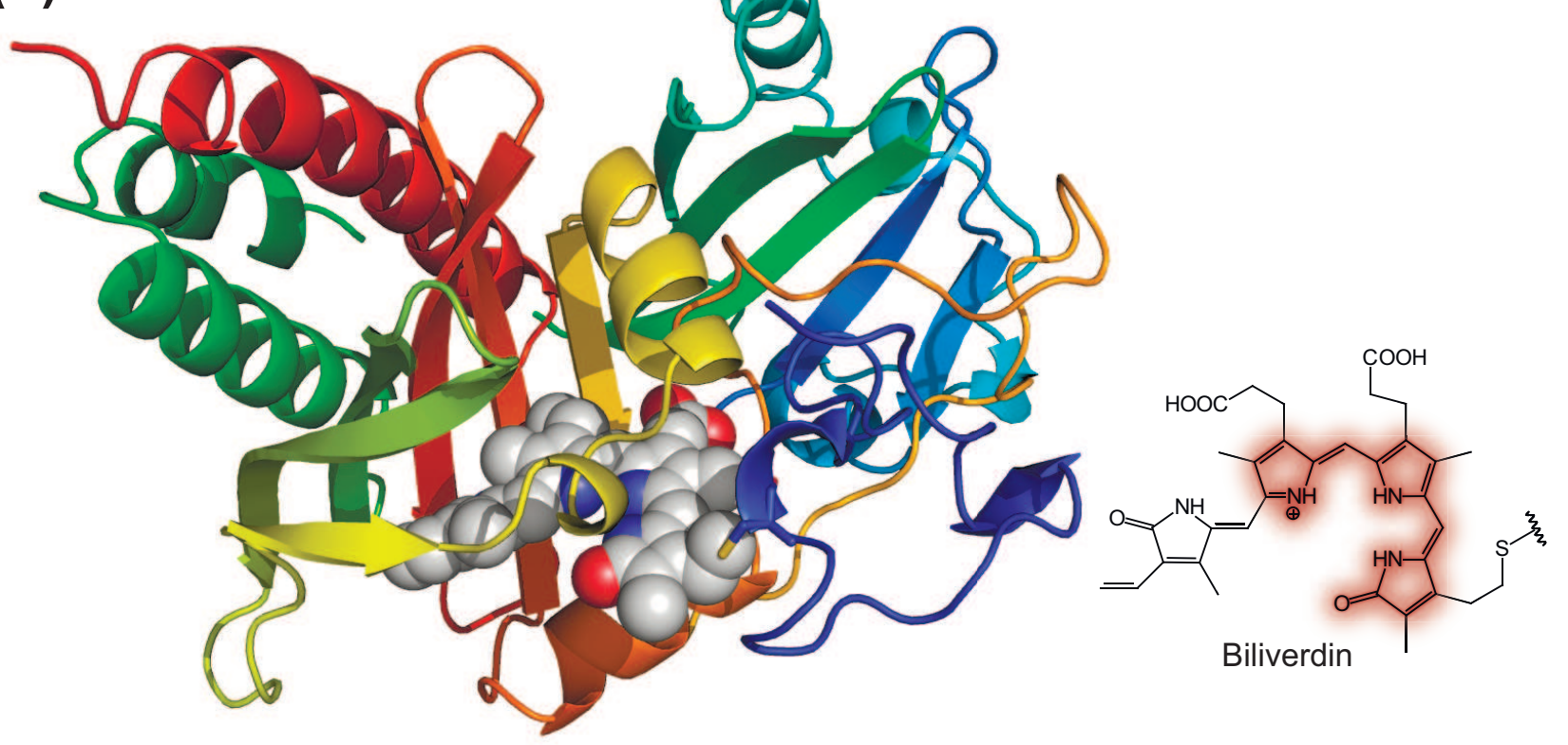


